\title{
A Systematic Review and Meta-Analysis of the SuperPATH Approach in Hip Arthroplasty
}

\author{
Yanzhi Ge $\mathbb{D}^{1},{ }^{1}$ Zuxiang Chen $\left(\mathbb{D},{ }^{1}\right.$ Qisong Chen $\mathbb{D}^{2},{ }^{2}$ Yanbin Fu $\mathbb{D}^{\circ},{ }^{3}$ Mengqiang Fan $\left(\mathbb{D},{ }^{1}\right.$ \\ Ting Li $(1),{ }^{4}$ Letian Shan $\left(\mathbb{D},{ }^{1}\right.$ Peijian Tong $\left(\mathbb{1},{ }^{1}\right.$ and Li Zhou $\mathbb{1}^{1}$ \\ ${ }^{1}$ The First Affiliated Hospital, Zhejiang Chinese Medical University, Hangzhou, 310053 Zhejiang, China \\ ${ }^{2}$ Department of Clinical Pharmacy, Ningbo Yinzhou No.2 Hospital, Ningbo, 315400 Zhejiang, China \\ ${ }^{3}$ Guangdong Provincial People's Hospital, Guangdong Academy of Medical Sciences, School of Medicine, South China University \\ of Technology, Guangzhou, 510080 Guangdong, China \\ ${ }^{4}$ The First Affiliated Hospital, College of Medicine, Zhejiang University, Hangzhou, 310003 Zhejian, China
}

Correspondence should be addressed to Letian Shan; letian.shan@zcmu.edu.cn, Peijian Tong; tongpeijian@163.com, and Li Zhou; zhouli0117@foxmail.com

Received 8 April 2021; Accepted 6 July 2021; Published 22 July 2021

Academic Editor: Tao Huang

Copyright (c) 2021 Yanzhi Ge et al. This is an open access article distributed under the Creative Commons Attribution License, which permits unrestricted use, distribution, and reproduction in any medium, provided the original work is properly cited.

\begin{abstract}
Objective. To compare the clinical and radiographic results of the supercapsular percutaneously assisted total hip (SuperPATH) approach and the conventional approach in hip arthroplasty. Design. Based on a prepublished protocol (PROSPERO: CRD42020177717), we searched PubMed, Embase, and Cochrane for relevant literatures up to January 30, 2021. The methodological qualities were assessed using the guidelines provided by the Cochrane Collaboration for Systematic Reviews. Randomized- or fixed-effect models were used to calculate the weighted mean difference (WMD) or odds ratio (OR), respectively, for continuous and dichotomous variables. Results. 6 articles were included in the study, and 526 patients were selected, which included 233 cases in the SuperPATH groups and 279 cases in the conventional groups, and 4 cases performed two surgeries in succession. The SuperPATH group demonstrated shorter incision length (WMD $=-7.87,95 \%$ CI $(-10.05$, $-5.69), P<0.00001)$, decreased blood transfusion rate $(\mathrm{OR}=0.48,95 \% \mathrm{CI}(0.25,0.89), P=0.02)$, decreased visual analogue scale (VAS) $(\mathrm{WMD}=-0.40,95 \% \mathrm{CI}(-0.72,-0.08), P=0.02)$, and higher Harris hip score (HHS) $(\mathrm{WMD}=1.98,95 \%$ CI $(0.18,3.77)$, $P=0.03)$ than the conventional group. However, there was no difference in VAS $(P=0.14)$ and HHS $(P=0.86)$ between the two groups 3 months later, nor in the acetabular abduction angle $(P=0.32)$ in either group. Conclusions. SuperPATH, as a minimally invasive approach with its reduced tissue damage, quick postoperative recovery, and early rehabilitation, demonstrates the short-term advantages of hip arthroplasty. As the evidences in favor of the SuperPATH technique were limited in a small number of studies and short duration of follow-up, more research is required to further analyze its long-term effect.
\end{abstract}

\section{Introduction}

Hip arthroplasty is an effective method to manage various hip diseases. This operation is increasingly favored by surgeons because it relieves pain effectively and is associated with early mobilization and improved life quality among patients. In recent years, artificial joint replacement and surgical instruments have evolved rapidly. However, previous studies reported high risks of trauma, blood loss, and postoperative complications during the process of traditional approach [1]. The advancement in minimally invasive sur- gery technology has improved the surgical results and reduced the possibility of surgical injury. An increasing number of researchers are proposing and developing minimally invasive techniques as the trend of future surgical management [2-4].

The supercapsular percutaneously assisted total hip (SuperPATH) technology, first reported by Dr. Chow in September 2011, runs between the gluteus minimus and piriformis [5] without cutting off any hip muscles and therefore preserves the integrity of muscles surrounding the joint capsule [6]. As a minimally invasive technique, SuperPATH 
is conducive to rapid postsurgery rehabilitation of patients. Compared to the conventional approach, the SuperPATH technology is appreciated by surgeons for its small surgical incision, less soft tissue damage, and fast postoperative rehabilitation. Contrarily, Rasuli and Gofton [7] reported that SuperPATH demonstrated a longer learning curve and the proficiency continued to decrease beyond the first 50 cases, which potentially prolonged the operation time.

Herein, we performed several randomized controlled trials (RCTs) and clinical controlled trials (CCTs) to compare the SuperPATH approach with the conventional approach for hip arthroplasty. Through this systemic review and meta-analysis, we further explored the short-term curative outcomes of the SuperPATH approach.

\section{Methods}

Literature selection, assessments of eligibility criteria, and data extraction and analyses were performed based on the protocol registered in (PROSPERO: CRD42020177717).

2.1. Search Strategies. A computerized retrieval of relevant literatures from PubMed, Embase and Cochrane library were performed up to January 30, 2021 to identify qualified trials and studies regarding SuperPATH. All aspects of the international systematic review were followed using the Cochrane handbook and the study was written according to the Preferred Reporting Items for Systematic Reviews and Meta-Analyses (PRISMA) statement [8]. Keywords related to SuperPATH and the medical subject headings $(\mathrm{MeSH})$ related to hip osteoarthritis, femoral neck fractures, femoral head necrosis and hip arthroplasty were used. The search strategy in PubMed was shown in Table 1, and the search strategy for the other two databases was omitted due to the similar steps.

2.2. Inclusion and Exclusion Criteria. Only RCTs, prospective or retrospective comparative studies, were enrolled. Inclusion criteria were (1) degenerative hip arthritis, avascular necrosis of the femoral head, or fresh femoral neck fractures; (2) SuperPATH approach or conventional approach (posterior approach, posterolateral approach, and direct anterior approach); and (3) having at least one of the following items reported: skin-to-skin operation time, length of incision, blood loss, blood transfusion rate, hospitalization time, visual analogue score (VAS), Harris hip score (HHS), and imaging measurements (acetabular anteversion angle and acetabular abduction angle).

Patients were removed for any of the following conditions: (1) revision of artificial total hip joint, severe osteoporosis, bone tumors, and muscle or nervous system diseases; (2) no comparison of the two approaches; (3) follow-up period of less than a year; and (4) republished literature, case reports, and reviews.

2.3. Data Extraction and Quality Assessment. Two reviewers were independently in accordance with the inclusion criteria for the quality of the literature evaluation and data extraction and then cross-checked. If there were any disagreements between them, a senior (Peijian Tong) would make a decision. RCTs were evaluated for quality using
TABle 1: PubMed search strategy.

\begin{tabular}{|c|c|}
\hline Sequence & Command search \\
\hline 1 & $\begin{array}{c}\text { \#1 “clinical trial” }[\mathrm{PT}] \text { OR "follow up study" }[\mathrm{PT}] \mathrm{OR} \\
\text { "randomized controlled trial”[PT] OR "cohort } \\
\text { study" }[\mathrm{PT}]\end{array}$ \\
\hline 2 & $\begin{array}{c}\# 2 \text { ((( cohort[TIAB] }) \text { OR randomized[TIAB] }) \text { OR } \\
\text { randomly[TIAB] }) \text { OR trial } *[\text { TIAB] }]) \text { OR } \\
\text { placebo }[\text { TIAB }]\end{array}$ \\
\hline 3 & \#3 \#1 OR \#2 \\
\hline 4 & \#4 gonarthrosis[TIAB] \\
\hline 5 & \#5 “Osteoarthritis, Hip”[Mesh] \\
\hline 6 & \#6 “Femoral Neck Fractures”[Mesh] \\
\hline 7 & \#7 “Femur Head Necrosis”[Mesh] \\
\hline 8 & \#8 \#4 OR \#5 OR \#6 OR \#7 \\
\hline 9 & $\# 9$ approach $*[$ TIAB $]$ \\
\hline 10 & \#10 SuperPATH[TIAB] \\
\hline 11 & $\begin{array}{l}\text { \#11 supercapsular percutaneously assisted total } \\
\text { hip[TIAB] }\end{array}$ \\
\hline 12 & \#12 \#9 OR \#10 OR \#11 \\
\hline 13 & \#13 “Arthroplasty, Replacement, Hip”[Mesh] \\
\hline 14 & $\# 14$ \#3 AND \#8 AND \#12 AND \#13 \\
\hline
\end{tabular}

the Cochrane risk assessment tool [9]. The NewcastleOttawa Scale (NOS) [10] was used for the quality assessment of cohort studies. This scale includes three major parts, being the selection of study groups, ascertainment of exposure, and outcome as well as group comparability. The general scores greater than or equal to 7 were considered low risk of bias.

The following information was extracted from the study: (1) characteristics of the studies (author, published time, disease, surgery, type of study, case characteristics, etc.); (2) outcome indicators: skin-to-skin operation time, length of incision, blood loss, blood transfusion rate, hospitalization time, imaging measurements (acetabular anteversion angle and acetabular abduction angle), postoperative VAS, and HHS at 1 week, 1 month, 3 months, 6 months, and 1 year after surgery.

2.4. Statistical Analyses. Meta-analysis was performed with Revman5.3 (Cochrane Collaboration, Copenhagen, Denmark) software for the available indicators. For the dichotomous variable, the odds ratios (ORs) and 95\% confidence interval (95\% CI) were used as the effect indicators. For the continuous variables, the weighted mean difference (WMD) and $95 \%$ CI were used as the effect indicator. When statistical heterogeneity of the study did not exist $(P \geq 0.1$ or $\left.I^{2}<50 \%\right)$, the fixed-effect model was used. When statistical heterogeneity existed $\left(P<0.1\right.$ or $\left.I^{2}>50 \%\right)$, the randomeffect model was used for data synthesis. The results of this meta-analysis were shown in the forest plot, and $P<0.05$ was considered as statistically significant.

\section{Results}

3.1. Literature Search Results. A total of 392 articles were initially obtained, of which 127 articles were excluded for 


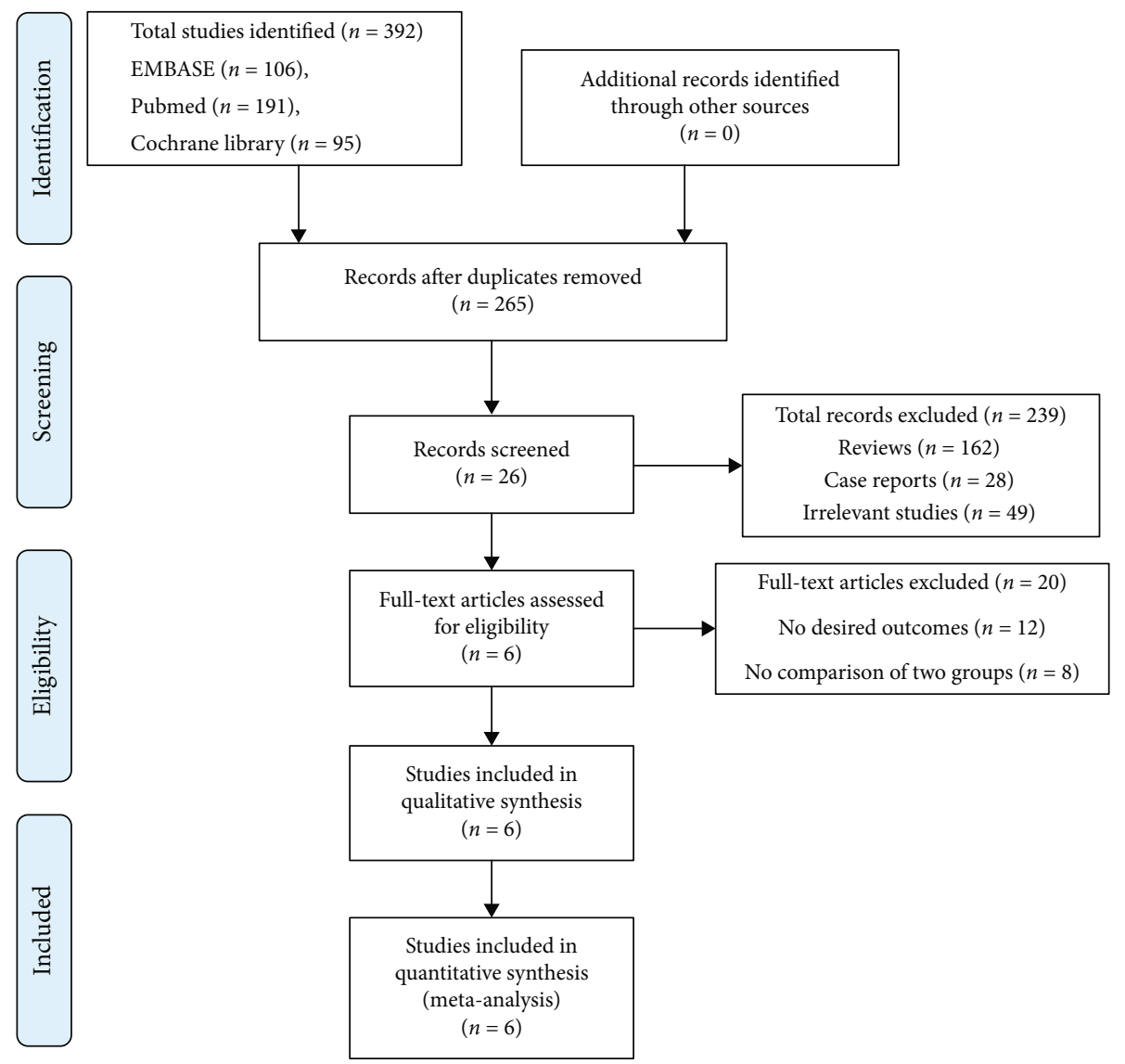

Figure 1: PRISMA study flow diagram.

repeated publishing. By reading titles and abstracts, 239 articles were excluded from reviews, case reports, and irrelevant studies. For no comparison of two approaches and literature that was not related to the purpose of the study, 8 documents were screened out. Further checking the context led to the exclusion of 9 articles lacking required data and 3 articles including other irrelevant interventions. Finally, 6 qualified articles [11-16] were selected. The literature inclusion and exclusion processes were shown in Figure 1, following the PRISMA statement.

3.2. The Basic Characteristics and Quality Evaluation of Included Studies. The basic characteristics of the 526 patients were shown in Table 2. Concerning the body mass index, one of the studies [16] was not mentioned. Regarding RCTs, there were a total of 3 studies $[11,12,16]$ enrolled in this article. Due to the particularity of surgery, all RCT studies did not report the methods of binding to participants and/or operators. Besides, one paper [11] explained the randomization but no randomized paired design was mentioned. None of the 3 RCTs had incomplete outcome or detection bias. The risk of bias graph and summary for the RCT studies were all shown in Figures 2 and 3. As shown in Table 3, the NOS scores of these three cohort studies [13-15] were 8, 8, and 8. In general, all of the 6 articles included in this study were of good quality, with standardized research design and good research value. The number of studies enrolled was less than 10; therefore, no publication bias was conducted.

\subsection{Surgical Outcomes}

3.3.1. Operation Time. Operation time (Figure 4(a)) was recorded in 6 studies [11-16] and random effects models were used because of the statistical heterogeneity of the results $\left(\chi^{2}=44.29, \mathrm{df}=5, I^{2}=89 \%, \quad P<0.00001\right)$. The results showed that there was no significant difference between the two groups $(\mathrm{WMD}=6.81,95 \%$ CI $(-1.47$, 15.09), $P=0.11)$.

3.3.2. Incision Length. Based on the available data from 4 studies $[11,12,15,16]$, we found significant heterogeneity $\left(\chi^{2}=111.20, \mathrm{df}=3, I^{2}=97 \%, P<0.00001\right)$. As was shown in Figure 4(b), the pooled results were statistically different between the two groups based on the random-effect model (WMD $=-7.87,95 \%$ CI $(-10.05,-5.69), P<0.00001)$.

3.3.3. Intraoperative Blood Loss. 6 studies [11-16] recorded intraoperative blood loss. Because of statistical heterogeneity $\left(\chi^{2}=402.99, \mathrm{df}=5, I^{2}=99 \%, P<0.00001\right)$, random-effect models were applied. The results in Figure 4(c) showed no significant difference between the two groups $(\mathrm{WMD}=$ 41.74, 95\% CI $(-53.22,136.70), P=0.39)$. 


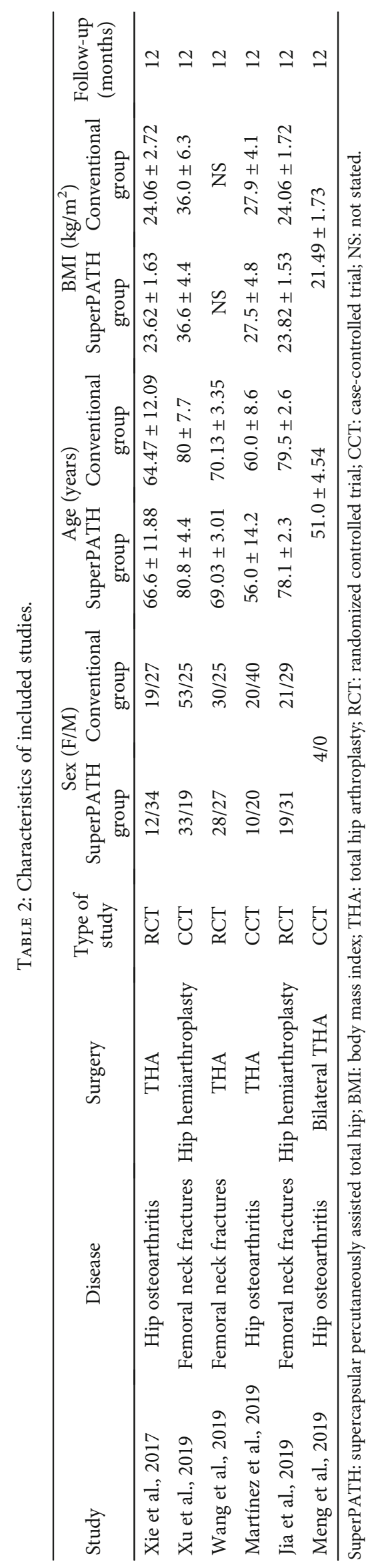




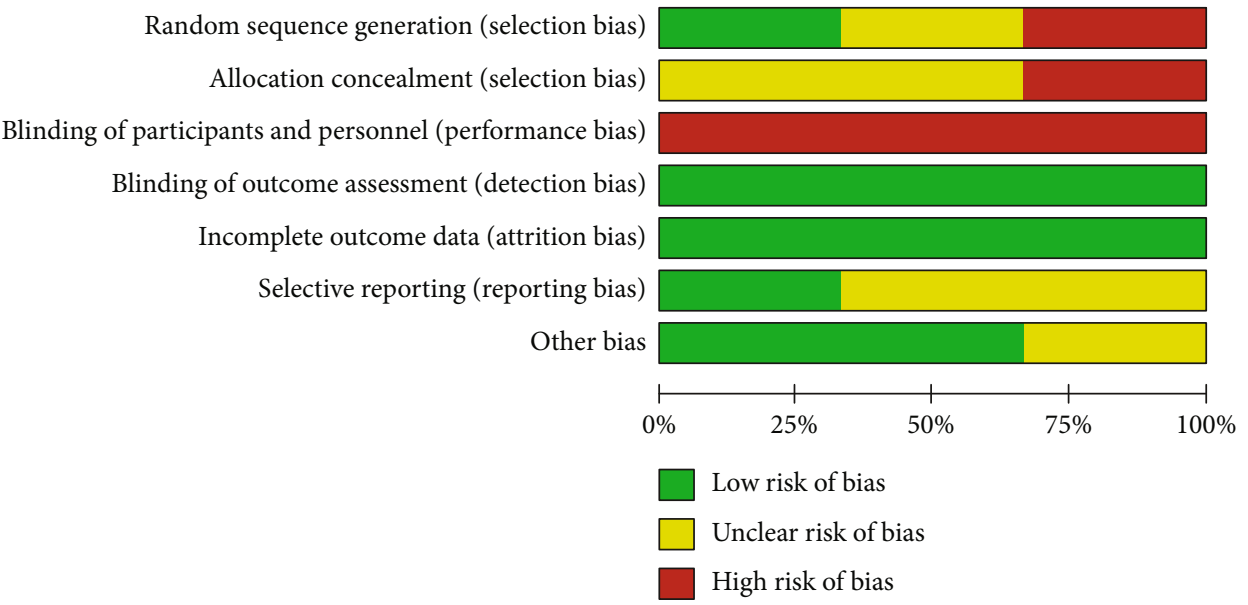

FIGURE 2: Risk of bias graph. Each risk of bias item is presented as percentages across all included studies.
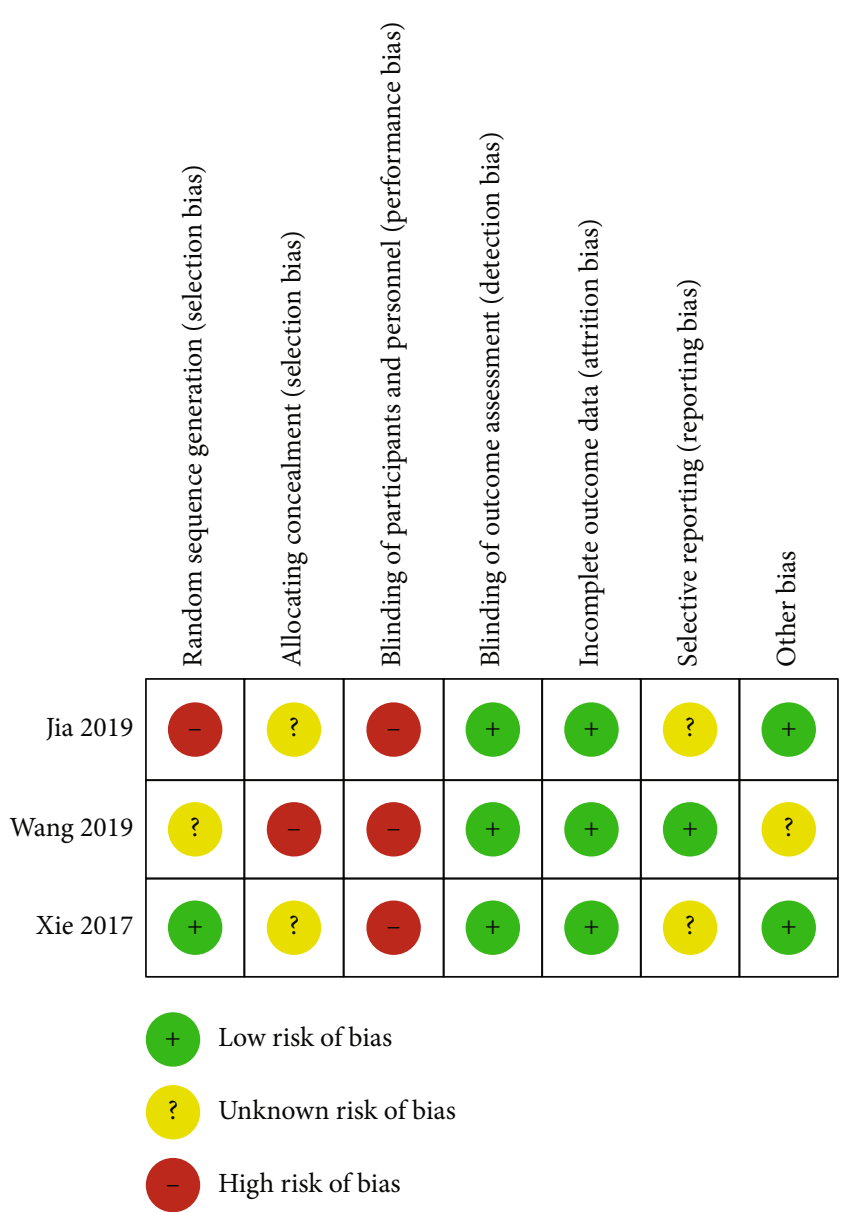

FIGURE 3: Risk of bias summary.

3.3.4. Blood Transfusion Rate. Data extracted from 4 studies $[11,12,14,15]$, including 326 participants, showed a postoperative transfusion rate (Figure 4(d)). Due to no significant differences in heterogeneity $\left(\chi^{2}=2.05, \mathrm{df}=2, I^{2}=2 \%, P=\right.$ 0.36 ), data was summarized using the fixed-effect model. The pooled results showed statistically significant difference between the two groups $(\mathrm{OR}=0.48,95 \% \mathrm{CI}(0.25,0.89)$, $P=0.01)$.

3.3.5. Hospitalization Time. A total of 206 participants (Figure 4(e)) referred to the length of stay $[11,15,16]$. Due to no significant differences in heterogeneity $\left(\chi^{2}=45.28\right.$, $\left.\mathrm{df}=2, I^{2}=96 \%, P<0.00001\right)$, data was summarized using random-effect models. The pooled results showed no statistically significant difference between the two groups $(\mathrm{WMD}=-1.94,95 \%$ CI $(-4.69,0.82), P=0.17)$.

3.4. Radiological Outcomes (Acetabular Anteversion Angle and Acetabular Abduction Angle). As was shown in Figure 4(f), two studies $[11,15]$ showed significant heterogeneity of the acetabular anteversion angle. The fixed-effect model was adopted $\left(\chi^{2}=1.70, \mathrm{df}=1, I^{2}=41 \%, P=0.19\right)$. There was significant difference between the two groups (WMD $=-0.98,95 \%$ CI $(-1.6,-0.31), P=0.004)$.

As was shown in Figure 4(g), the results of two studies $[11,15]$ showed no statistically significant heterogeneity in the acetabular abduction angle $\left(\chi^{2}=1.06, \mathrm{df}=1, I^{2}=6 \%\right.$, $P=0.30$ ). The difference between the two groups was no statistically significant $(\mathrm{WMD}=-1.32,95 \%$ CI $\quad(-3.92$, 1.27), $P=0.32$ ).

\subsection{Functional Outcomes}

3.5.1. VAS. 4 articles $[11,12,15,16]$ mentioned VAS (Figure 5(a)). We found significant heterogeneity in pooled results, so we used the random-effect model $\left(\chi^{2}=282.54\right.$, $\left.\mathrm{df}=13, I^{2}=95 \%, P<0.00001\right)$. The pooled follow-up results showed a statistically significant difference between the two groups (WMD $=-0.40,95 \% \mathrm{CI}(-0.72,-0.08), P=0.02)$.

The subgroup analysis of VAS at 1 week and $1,3,6$, and 12 months after operation showed that week 1 (WMD = $-1.33,95 \%$ CI $(-2.16,-0.51), P=0.02)$ was statistically significant but indicated no statistical significance at one month (WMD $=-0.46,95 \%$ CI $(-1.12,0.20), P=0.17)$, three months (WMD $=-0.17,95 \%$ CI $(-0.41,0.06), P=$ 0.14 ), six months (WMD $=-0.05,95 \%$ CI $(-0.23,0.14)$, 
TABle 3: Newcastle-Ottawa Scale for risk of bias assessment of cohort studies included in the meta-analysis.

\begin{tabular}{|c|c|c|c|c|c|c|c|c|c|}
\hline \multirow{2}{*}{ Study } & \multicolumn{4}{|c|}{ Selection } & \multirow{2}{*}{ Comparability } & \multicolumn{3}{|c|}{ Outcome } & \multirow{2}{*}{ Overall scores } \\
\hline & 1 & 2 & 3 & 4 & & 5 & 6 & 7 & \\
\hline Martínez et al. & $\star$ & $\star$ & $\star$ & $\star$ & $\star \star$ & is & $\star$ & $\star$ & 8 \\
\hline $\mathrm{Xu}$ et al. & $\star$ & $\star$ & $\star$ & $\star$ & $\star \star$ & $\star$ & $\star$ & is & 8 \\
\hline Meng et al. & is & $\star$ & $\star$ & $\star$ & $\star \star$ & $\star$ & $\star$ & $\star$ & 8 \\
\hline
\end{tabular}

1: representativeness of exposed cohort; 2 : selection of nonexposed; 3: ascertainment of exposure; 4: outcome not present at start; 5 : assessment of outcome; 6 : adequate follow-up length; 7 : adequacy of follow-up. $\lesssim$ score of $0, \star$ score of $1, \star \star$ score of 2 .

$P=0.62)$, and a year $(\mathrm{WMD}=-0.09,95 \%$ CI $(-0.21$, $0.02), P=0.12)$ after surgery.

3.5.2. HHS. As was shown in Figure 5(b), the statistical analysis of 5 studies $[11-13,15,16]$ showed statistically significant heterogeneity $\left(\chi^{2}=368.59\right.$, $\mathrm{df}=16, I^{2}=96 \%, P<$ $0.00001)$. The difference of pooled follow-up results between two groups was statistically significant $(\mathrm{WMD}=1.98,95 \% \mathrm{CI}$ $(0.18,3.77), P=0.03)$.

The meta-analysis of HHS showed that the two groups were significantly different at 1 week after surgery (WMD $=7.96,95 \%$ CI $(3.63,12.28), P=0.0003), 1$ month after operation $(\mathrm{WMD}=3.76,95 \%$ CI $(2.09,5.42), P<$ $0.00001)$, and a year after operation $(\mathrm{WMD}=0.84,95 \% \mathrm{CI}$ $(0.17,1.51), P=0.01)$, but not at three months $(\mathrm{WMD}=$ $-0.33,95 \%$ CI $(-4.15,3.49), P=0.86)$ nor six months $(\mathrm{WMD}=-0.09,95 \% \mathrm{CI}(-1.73,1.55), P=0.91)$ after surgery.

\section{Discussion}

The SuperPATH technology is a combination of the SuperCap technique [5] and PATH technique [17] and has become a revolutionary approach with minimal invasion. It is consistent with the anatomical landmarks of the conventional posterolateral approach, as it preserves all the benefits of the standard posterolateral technique, such as the clearly exposed vision and precision of prosthesis installation. Also, the SuperPATH technology does not require special operative tables and can be easily converted to a standard posterior approach. During soft tissue separation, the SuperPATH technology does not transversely cutoff muscles and tendons, which preserves the integrity of the adjacent external rotators to the joint capsule. Furthermore, acetabular preparation is straightforward, aided by the percutaneous incision, thereby providing a rapid postoperative recovery as the muscles remain intact. A previous meta-analysis [18] revealed the advantage of SuperPATH approach sourcing mostly from Chinese database and literatures, which was quite different from our study.

In the current study, we performed a systematic review and meta-analysis that compared the SuperPATH approach with the conventional approach in hip arthroplasty. 6 studies (3 RCTs and 3 CCTs) met our inclusion criteria. In terms of incision length, four studies reported a shorter average length of the surgical incision in the SuperPATH group than that in the conventional group. Unlike the great trauma caused by the conventional approach, minimally invasive surgery brings a series of advantages which cannot be defined by short incision only. For instance, direct anterior access may cause lateral femoral cutaneous nerve injury [19], whereas the lateral approach may cause the superior gluteal nerve injury and cutoff the insertion of the gluteus minimus [20]. Furthermore, the posterior lateral approach requires incising the piriformis muscle to expose the joint capsule [21]. As a minimally invasive surgery, SuperPATH is a satisfactory approach not only for its minimal incision but also for its protection of the external muscles and ligaments without cutting them off. We found that violent pulling of the distal or proximal soft tissue during surgery potentially reduced postoperative incision necrosis, subcutaneous fat liquefaction, and other complications associated with other minimally invasive THAs. Thus, obesity and stiffness of muscles are contraindications for conventional surgical options. On account of a short incision, mini-invasive surgery may be linked to the inaccuracy of prosthesis placement due to the missing field of vision and unclear anatomical landmarks. However, SuperPATH is not a contraindication to obesity and stiffness of muscles. This further broadens the indications for the resolution of intraoperative joint capsule exposures and anatomic landmarks [3]. In another study, Eskelinen [22] suggested that more attention should be given to tissue protection and placement of the prosthesis and the short incision $(\leq 10 \mathrm{~cm})$ should be made into the secondary site simultaneously. Elsewhere, Han et al. [23] found that intraoperative protection of the external rotation muscles could lower the postoperative dislocation rate from $1.8 \%$ to $6.2 \%$.

In the present study, 6 articles documented the operation time. The results revealed no difference in the operating time between the two groups, which may be closely associated with the learning curve of the new SuperPATH technology. For instance, Rasuli and Gofton [7] reported 50 cases and 49 cases adopting SuperPATH and PATH, respectively, as minimally invasive approaches for total hip arthroplasty. In their study, the operation time of the SuperPATH group exhibited a decreasing trend, suggesting that the learning curve may be associated with prolonged operation time. Besides, the incision suturing step was, in most cases, performed by junior physicians at the end of the surgery and may impact the overall operation time.

Blood loss and transfusion rates in prosthetic hip operations were closely associated with the bleeding during osteotomy, intramedullary reaming of the medullary cavity, and muscle excision. With the SuperPATH approach, smaller soft tissue dissection was associated with less bleeding and lower transfusions rates. In our analysis, 6 studies and 4 


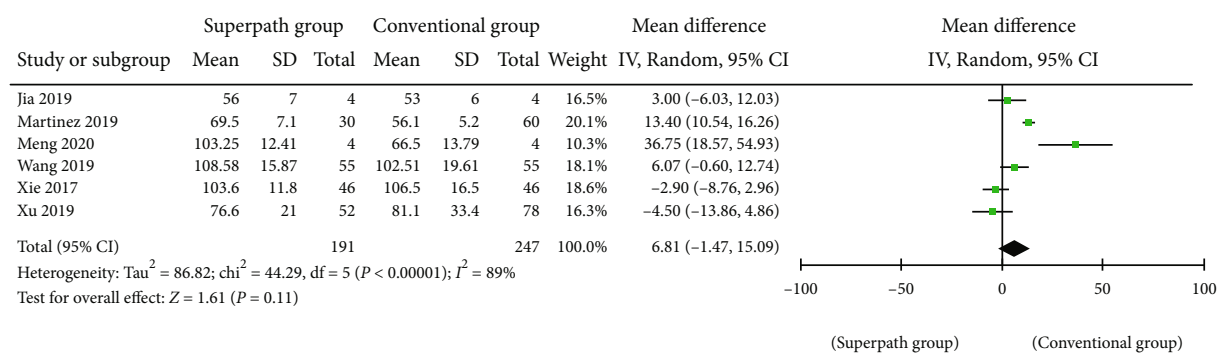

(a)

\begin{tabular}{|c|c|c|c|c|c|c|c|c|c|c|c|c|}
\hline \multirow{3}{*}{$\begin{array}{l}\text { Study or subgroup } \\
\text { Jia } 2019\end{array}$} & \multicolumn{3}{|c|}{ Superpath group } & \multicolumn{3}{|c|}{ Conventional group } & \multicolumn{3}{|c|}{ Mean difference } & \multirow{2}{*}{\multicolumn{2}{|c|}{$\begin{array}{c}\text { Mean difference } \\
\text { IV, Random, 95\% CI }\end{array}$}} & \\
\hline & \multirow{2}{*}{$\begin{array}{r}\text { Mean } \\
7.1\end{array}$} & \multirow{2}{*}{$\frac{\mathrm{SD}}{0.6}$} & \multirow{2}{*}{$\begin{array}{r}\text { Total } \\
50\end{array}$} & \multirow{2}{*}{$\begin{array}{r}\text { Mean } \\
17.1\end{array}$} & \multirow{2}{*}{$\begin{array}{r}\mathrm{SD} \\
1.9\end{array}$} & \multirow{2}{*}{$\begin{array}{r}\text { Total } \\
50\end{array}$} & \multirow{2}{*}{$\begin{array}{r}\text { Weight } \\
25.7 \%\end{array}$} & \multirow{2}{*}{$\frac{\text { IV, Random, } 95 \% \text { CI }}{-10.00(-10.55,-9.45)}$} & & & & \\
\hline & & & & & & & & & & $=$ & & \\
\hline Meng 2020 & 7.62 & 0.97 & 4 & 11.12 & 1.21 & 4 & $23.2 \%$ & $-3.50(-5.02,-1.98)$ & & $\rightarrow$ & & \\
\hline Wang 2019 & 6.65 & 1.53 & 55 & 17.08 & 1.4 & 55 & $25.7 \%$ & $-10.43(-10.98,-9.88)$ & & $=$ & & \\
\hline Xie 2017 & 7.4 & 1.06 & 46 & 14.5 & 2.38 & 46 & $25.4 \%$ & $-7.10(-7.85,-6.35)$ & & $=$ & & \\
\hline Total $(95 \% \mathrm{CI})$ & & & 155 & & & 155 & $100.0 \%$ & $-7.87(-10.05,-5.69)$ & & 0 & & \\
\hline $\begin{array}{l}\text { Heterogeneity: } \mathrm{Tau}^{2}=4 \\
\text { Test for overall effect: } Z\end{array}$ & $\begin{array}{l}.73 ; \mathrm{chi}^{2}= \\
=7.07(P\end{array}$ & $\begin{array}{l}111.20, \\
0.0000\end{array}$ & $\begin{array}{l}\mathrm{df}=3(1 \\
\mathrm{d})\end{array}$ & $P<0.000$ & $I^{2}=$ & & & & -20 & -10 & 10 & 20 \\
\hline
\end{tabular}

(b)

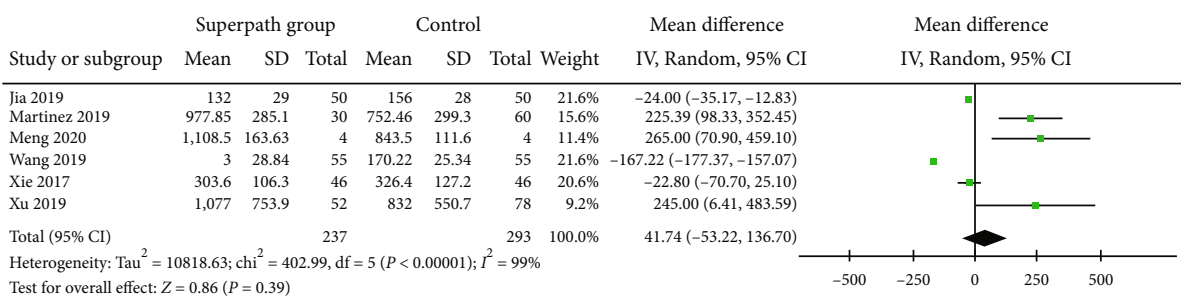
Test for overall effect: $Z=0.86(P=0.39)$

(c)

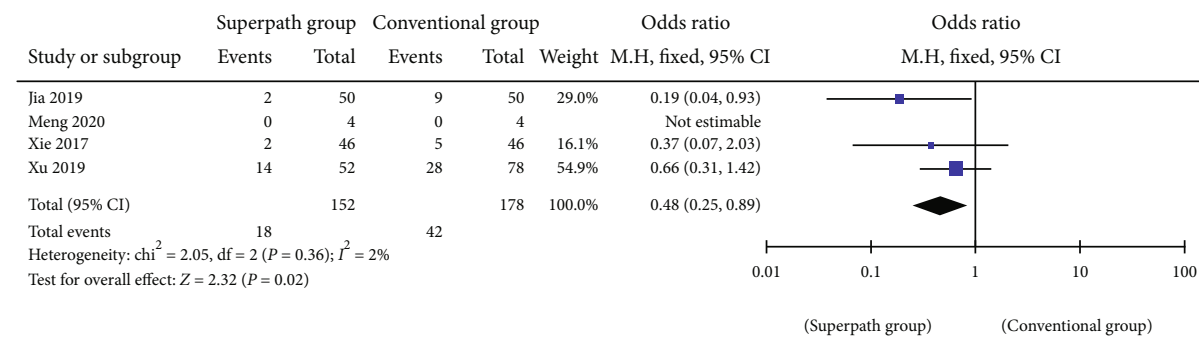

(d)

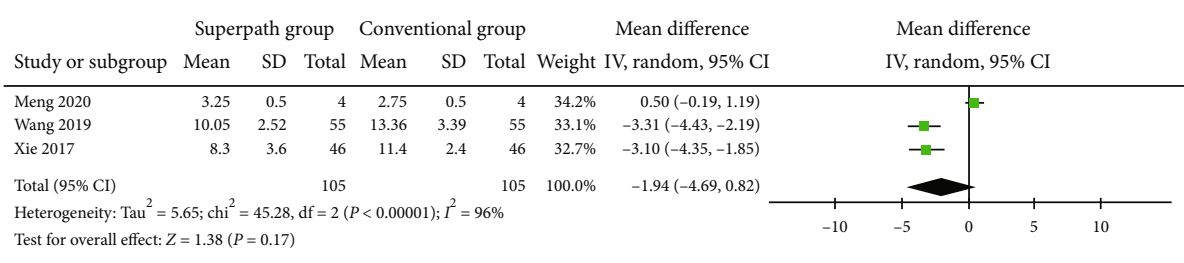

(e)

\begin{tabular}{|c|c|c|c|c|c|c|c|c|c|c|c|c|c|}
\hline \multirow[b]{2}{*}{ Study or subgroup } & \multicolumn{3}{|c|}{ Superpath group } & \multicolumn{3}{|c|}{ Conventional group } & & \multirow{2}{*}{$\begin{array}{l}\text { Mean difference } \\
\text { IV, Fixedf, 95\% CI }\end{array}$} & & \multicolumn{3}{|c|}{ Mean difference } & \\
\hline & Mean & SD & Total & Mean & SD & Total & Weight & & & & & d, $95 \%$ CI & \\
\hline Meng 2020 & 15 & 1.82 & 4 & 14.25 & 2.06 & 4 & $6.3 \%$ & $0.75(-1.94,3.44)$ & & & & & \\
\hline Xie 2017 & 17.4 & 1.6 & 46 & 18.5 & 1.8 & 46 & $93.7 \%$ & $-1.10(-1.80,-0.40)$ & & & & & \\
\hline Total $(95 \% \mathrm{CI})$ & & & 50 & & & 50 & $100.0 \%$ & $-0.98(-1.66,-0.31)$ & & & & & \\
\hline $\begin{array}{l}\text { Heterogeneity: chi }{ }^{2}=1 \\
\text { Test for overall effect: } Z\end{array}$ & $\begin{array}{l}.70, \mathrm{df}=1 \\
=2.86(P\end{array}$ & $\begin{array}{l}P=0.1 \\
=0.004\end{array}$ & 9); $I^{2}=$ & & & & & & -4 & -2 & o & 2 & 4 \\
\hline
\end{tabular}

(f)

FIgURE 4: Continued. 


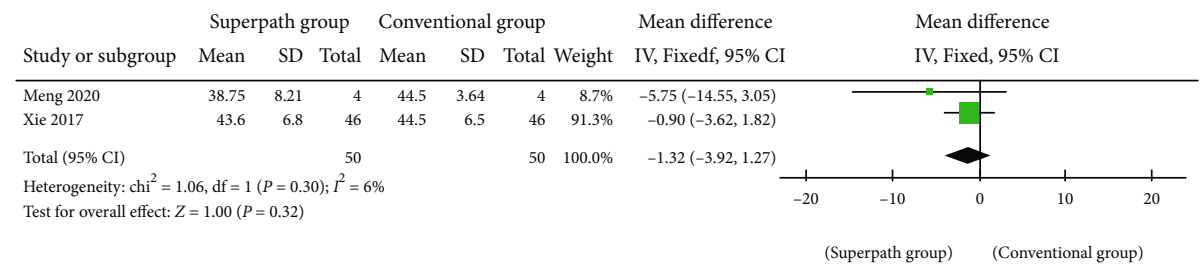

(g)

FIgURE 4: Forest plot analysis comparing the SuperPATH group vs the conventional group. (a) Operation time. (b) Incision length. (c) Blood loss. (d) Transfusion rate. Blood loss. (e) Hospitalization time. (f) Anteversion angle. (g) Abduction angle.

studies mentioned intraoperative blood loss and blood transfusion rates, respectively. Although no significant difference was found in intraoperative blood loss, blood transfusion rates were significantly lower with the SuperPATH technique, which is most likely because the SuperPATH technique can result in less trauma by protecting the joint capsule and external rotation muscles. As a new technique, surgeons may prolong the operation time and have increased blood loss risks for unskilled operation. Gofton et al. [6] and Rasuli and Gofton [7] found that the transfusion rates of SuperPATH minimally invasive surgery were $3.3 \%$ and $4.0 \%$, respectively, lower compared to those with conventional approaches.

3 studies reported on the hospitalization time but exhibited no significant difference. Cardenas-Nylander et al. [24] found that the average length of stay for patients who underwent SuperPATH surgery was 1.4 days, less than that of the conventional THA. Gofton and Fitch [25] explored the 30-day readmission rates for 479 patients who underwent THA and found that the 30-day readmission rate for the SuperPATH minimally invasive total hip arthroplasty ranged from $4.3 \%$ to $2.3 \%$ compared to conventional procedures. The same research team investigated 99 patients between April 2013 and January 2014 and reported that the overall costs of hospitalization of the SuperPATH approach were $28.4 \%$ lower than those of the conventional lateral approach [6]. Thus, SuperPATH significantly reduced postoperative costs. Meng et al. [15] in their study performed bilateral hip arthroplasty in succession, demonstrating that the SuperPATH approach could reduce the whole length of stay and enhance the postsurgery recovery.

4 studies mentioned VAS as well as the subgroup analysis revealed that 1 week after surgery, pain relief was significantly improved in the SuperPATH group than that in the conventional surgery group. However, there was no difference between the two groups during the 1 to 12 months follow-up. Based on the above discussion, the SuperPATH technology improved patient satisfaction in the early postoperative period. Bodrogi et al. [26] performed the SuperPATH technique in 17 patients with femoral neck fractures, and the clinical follow-up found that postoperative analgesia dosage was reduced and the hospital stays were shortened. Jiang [27] measured the circumference of the thigh after the SuperPATH surgery and found that the postoperative degree of edema was significantly lower than other small incision surgeries, thus reducing the patient pain and other constrained symptoms.
HHS is a widely used synthetical mark to evaluate the hip function and assess the effect of hip replacement. In this meta-analysis, the HHS at week 1 and one month postoperatively in the SuperPATH group was higher than that in the conventional replacement group. However, we found no significant difference after 3 months. None of the included articles mentioned specific details of the HHS. The author believed that HHS in the initial period was low in the SuperPATH replacement group, demonstrating a nearly functional recovery. The difference in the middle period was not significant, but the overall follow-up was shorter. And the long-term efficacy still needed a follow-up assessment. Della et al. [3] collected postoperative imaging measurements in 66 patients and found that the early results in the SuperPATH group were superior to the conventional group. However, the long-term results needed further investigation.

Moreover, Lewinnek et al. [28] found that the dislocation rate for cup orientation with anteversion of $15 \pm 10$ degrees and lateral opening of $40 \pm 10$ degrees was $1.5 \%$, with respect to minimal risks of dislocation in the postoperative period. Herein, following the analysis of 2 studies, we found no difference in acetabular abduction angles by comparing the SuperPATH group to the conventional group. Simultaneously, the two groups showed significant differences in acetabular anteversion angles. In a previous study by Williams et al. [29], the transverse acetabular ligament was applied to establish the position of the acetabular locator. This method was suitable for both conventional and minimally invasive procedures. Therefore, reaming and placement of the acetabular component referring to the transverse acetabular ligament can significantly improve the accuracy of the prosthesis position. Rasuli and Gofton [7] also revealed that the reference to acetabular transverse ligament during operation could increase the accuracy of the prosthesis and reduce the rate of dislocation. In our review, only two studies and 50 patients were enrolled for analysis, which may restrain the accuracy of our judgment.

This article had some limitations: (1) the follow-up of each study and some of the evaluation indicators were inconsistent, (2) the analysis lacks detailed score data and the incidence of complications was not assessed, (3) only published papers were identified; thus, unpublished articles might influence the ultimate result, and (4) the SuperPATH technology was first reported in 2011, and multicenters, larger samples, and follow-up assessments are still needed to determine its long-term efficacy and complications. 


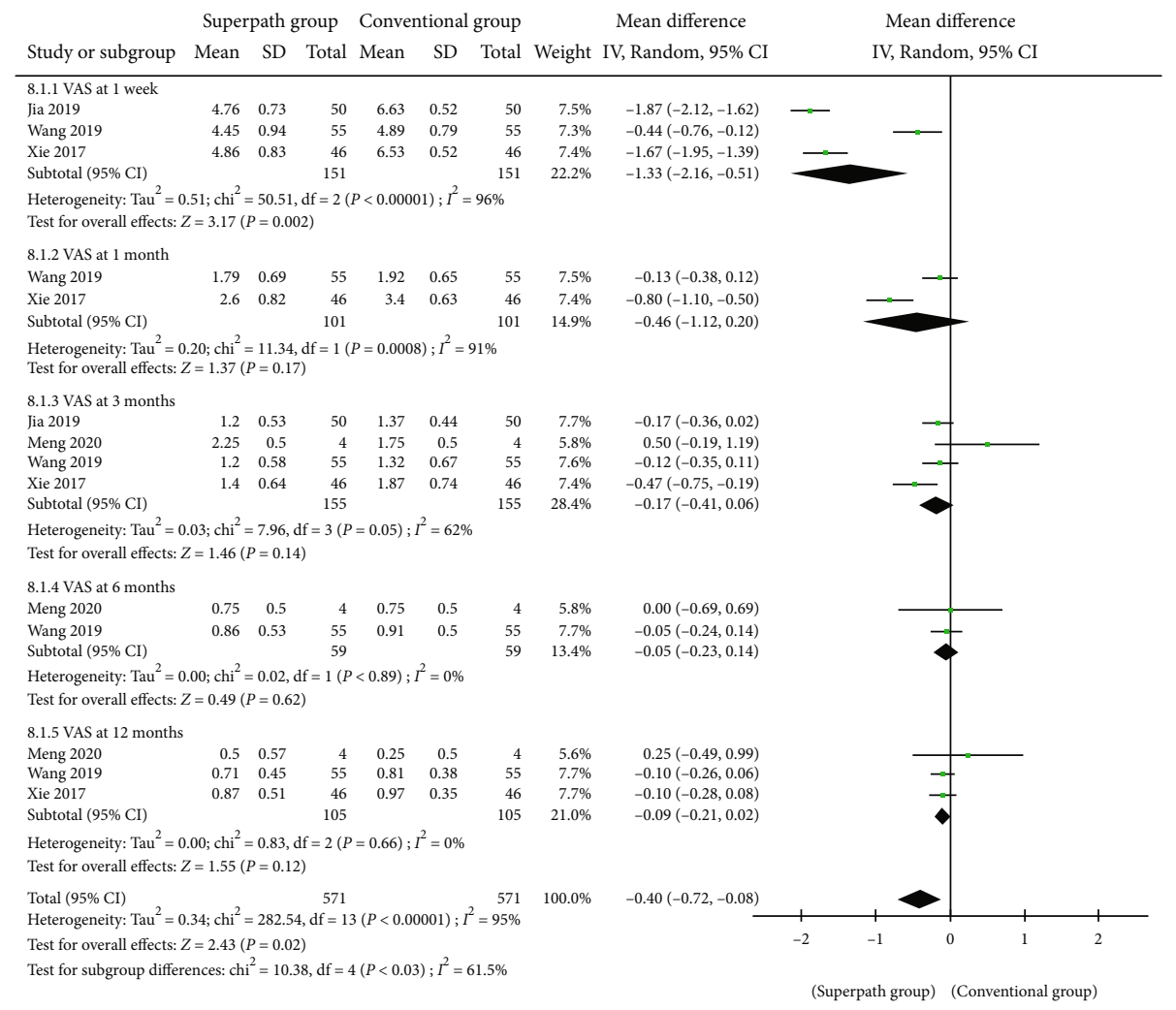

(a)

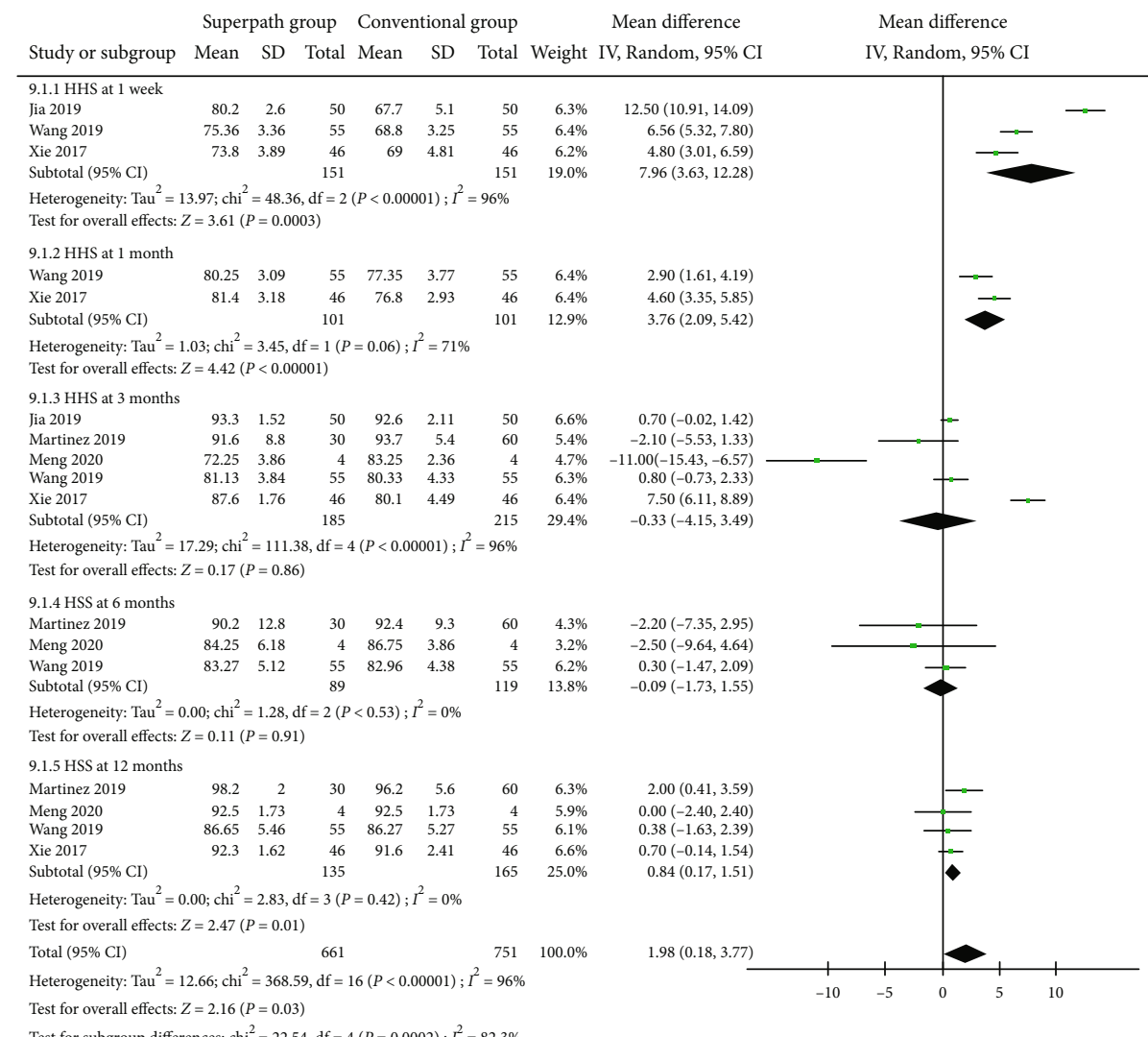

(b)

Figure 5: Forest plot analysis from 5 times comparing the SuperPATH group vs the conventional group. (a) VAS. (b) HHS. 
Our meta-analysis demonstrated that the short- and medium-term postoperative hip-related scores of the SuperPATH approach were not significantly different from those of the conventional approach. However, the SuperPATH approach, on account of shorter incision length, lower postoperative transfusion rate, preferable early postoperative VAS, and HHS, is superior to the conventional approach. Furthermore, it has potential benefits, including low tissue damage and rapid postoperative recovery, which can significantly improve the quality of life and satisfaction of patients.

\section{Conclusions}

Our study suggested that SuperPATH was associated with shorter incision length, less blood transfusion rate, better functional recovery, and lower pain scores. Due to the limited number of studies and insufficient sample sizes, the conclusion should be treated cautiously. And larger sample sizes with well-designed RCTs are required to confirm our conclusion.

\section{Data Availability}

All data can be obtained from corresponding author Li Zhou or the first author Yanzhi Ge.

\section{Disclosure}

This manuscript has been preprinted in Research Square (10.21203/rs.3.rs-41289/v1).

\section{Conflicts of Interest}

The authors declare that they have no conflicts of interest.

\section{Authors' Contributions}

The following authors designed the study (Yanzhi Ge, Peijian Tong), collected the data (Yanzhi Ge, Zuxiang Chen, and Yanbin Fu), analyzed the data (Li Zhou, Mengqiang Fan, and Ting Li), wrote the initial drafts (Yanzhi Ge), and ensured the accuracy of the data and analysis (Peijian Tong, Letian Shan, Li Zhou). Yanzhi Ge, Li Zhou, Qisong Chen, and Letian Shan revised the manuscript. All authors read and approved the manuscript. Yanzhi Ge and Zuxiang Chen contributed equally to this work.

\section{Acknowledgments}

The authors want to thank all patients and subjects for their participation. This work was supported by the scientific research project of the Zhejiang Education Commission (grant number Y202044541 to author Yanzhi Ge), Zhejiang Natural Science Foundation Young Scholars (grant number LQ20H270009 to author Li Zhou), Zhejiang Traditional Chinese Medical Science Foundation (grant number 2020ZA039 to author Li Zhou), and Natural Science Foundation of Zhejiang Chinese Medical University (grant number 2020ZG42 to author Li Zhou).

\section{References}

[1] X. Li, L. Ma, Q. Wang, and K. Rong, "Comparison of total hip arthroplasty with minimally invasive SuperPath approach vs. conventional posterolateral approach in elderly patients: a one-year follow-up randomized controlled research," Asian Journal of Surgery, vol. 44, no. 3, pp. 531-536, 2021.

[2] D. J. Roger and D. Hill, "Minimally invasive total hip arthroplasty using a transpiriformis approach: a preliminary report," Clinical Orthopaedics and Related Research, vol. 470, no. 8, pp. 2227-2234, 2012.

[3] P. K. Della Torre, D. A. Fitch, and J. C. Chow, "Supercapsular percutaneously-assisted total hip arthroplasty: radiographic outcomes and surgical technique," Ann Transl Med, vol. 3, no. 13, 2015.

[4] N. Capuano, A. Del Buono, and N. Maffulli, "Tissue preserving total hip arthroplasty using superior capsulotomy," Operative Orthopädie und Traumatologie, vol. 27, no. 4, pp. 334-341, 2015.

[5] J. Chow, B. Penenberg, and S. Murphy, "Modified microsuperior percutaneously-assisted total hip: early experiences \& case reports," Current Reviews in Musculoskeletal Medicine, vol. 4, no. 3, pp. 146-150, 2011.

[6] W. Gofton, J. Chow, K. D. Olsen, and D. A. Fitch, "Thirty-day readmission rate and discharge status following total hip arthroplasty using the supercapsular percutaneously-assisted total hip surgical technique," International Orthopaedics, vol. 39, no. 5, pp. 847-851, 2015.

[7] K. J. Rasuli and W. Gofton, "Percutaneously assisted total hip (PATH) and Supercapsular percutaneously assisted total hip (SuperPATH) arthroplasty: learning curves and early outcomes," Ann Transl Med, vol. 3, no. 13, p. 179, 2015.

[8] M. D. F. McInnes, D. Moher, B. D. Thombs et al., "Preferred reporting items for a systematic review and meta-analysis of diagnostic test accuracy studies: the PRISMA-DTA statement," JAMA, vol. 319, no. 4, pp. 388-396, 2018.

[9] J. P. T. Higgins, D. G. Altman, P. C. Gotzsche et al., "The Cochrane Collaboration's tool for assessing risk of bias in randomised trials," BMJ, vol. 343, no. oct18 2, p. d5928, 2011.

[10] A. Stang, S. Jonas, and C. Poole, "Case study in major quotation errors: a critical commentary on the Newcastle-Ottawa scale," European Journal of Epidemiology, vol. 33, no. 11, pp. 1025-1031, 2018.

[11] J. Xie, H. Zhang, L. Wang, X. Yao, Z. Pan, and Q. Jiang, “Comparison of supercapsular percutaneously assisted approach total hip versus conventional posterior approach for total hip arthroplasty: a prospective, randomized controlled trial," Journal of Orthopaedic Surgery and Research, vol. 12, no. 1, p. 138, 2017.

[12] J. Jianbo, J. Ying, L. Xinxin, W. Lianghao, Y. Baoqing, and A. Rongguang, "Hip hemiarthroplasty for senile femoral neck fractures: minimally invasive SuperPath approach versus traditional posterior approach," Injury, vol. 50, no. 8, pp. 1452-1459, 2019.

[13] J. Más Martínez, J. Sanz-Reig, M. Morales-Santías, D. Bustamante Suarez de Puga, C. Verdu Roman, and E. Martinez Gimenez, "Comparative cohort study of the SuperPath approach and the conventional posterior approach in primary cementless hip replacement surgery," Rev Esp Cir Ortop Traumatol (Engl Ed), vol. 63, no. 5, pp. 346-354, 2019.

[14] K. Xu, D. Anwaier, R. He et al., "Hidden blood loss after hip hemiarthroplasty using the superPATH approach: a retrospective study," Injury, vol. 50, no. 12, pp. 2282-2286, 2019. 
[15] W. Meng, Z. Huang, H. Wang et al., "Supercapsular percutaneously-assisted total hip (SuperPath) versus posterolateral total hip arthroplasty in bilateral osteonecrosis of the femoral head: a pilot clinical trial," BMC Musculoskeletal Disorders, vol. 21, no. 1, p. 2, 2019.

[16] X. D. Wang, H. Lan, Z. X. Hu et al., "SuperPATH minimally invasive approach to total hip arthroplasty of femoral neck fractures in the elderly: preliminary clinical results," Orthopaedic Surgery, vol. 12, no. 1, pp. 74-85, 2020.

[17] B. L. Penenberg, W. S. Bolling, and M. Riley, "Percutaneously assisted total hip arthroplasty (PATH): a preliminary report," The Journal of Bone and Joint Surgery. American Volume, vol. 90, Supplement_4, pp. 209-220, 2008.

[18] N. Ramadanov, S. Bueschges, K. Liu, R. Klein, and R. Schultka, "Comparison of short-term outcomes between SuperPATH approach and conventional approaches in hip replacement: a systematic review and meta-analysis of randomized controlled trials," Journal of Orthopaedic Surgery and Research, vol. 15, no. 1, p. 420, 2020.

[19] Y. Ozaki, Y. Homma, T. Baba, K. Sano, A. Desroches, and K. Kaneko, "Spontaneous healing of lateral femoral cutaneous nerve injury and improved quality of life after total hip arthroplasty via a direct anterior approach," Journal of Orthopaedic Surgery, vol. 25, no. 1, 2017.

[20] T. Khan and D. Knowles, "Damage to the superior gluteal nerve during the direct lateral approach to the hip: a cadaveric study," The Journal of Arthroplasty, vol. 22, no. 8, pp. 11981200, 2007.

[21] T. Wang, L. Shao, W. Xu, F. Li, and W. Huang, "Surgical injury and repair of hip external rotators in THA via posterior approach: a three-dimensional MRI-evident quantitative prospective study," BMC Musculoskeletal Disorders, vol. 20, no. 1, p. 22, 2019.

[22] A. Eskelinen, "Minimal incision total hip arthroplasty. A concise follow-up report on functional and radiographic outcomes at 10 years," Journal of Bone and Joint Surgery, vol. 99, no. 20, 2017.

[23] S. K. Han, Y. S. Kim, and S. H. Kang, "Treatment of femoral neck fractures with bipolar hemiarthroplasty using a modified minimally invasive posterior approach in patients with neurological disorders," Orthopedics, vol. 35, no. 5, pp. e635-e640, 2012.

[24] C. Cardenas-Nylander, V. Bellotti, E. Astarita, E. M. Gomez, and M. R. Fernandez, "Innovative approach in total hip arthroplasty: supercapsular percutaneously-assisted," Hip International, vol. 26, Supplement 1, 2016.

[25] W. Gofton and D. A. Fitch, "In-hospital cost comparison between the standard lateral and supercapsular percutaneouslyassisted total hip surgical techniques for total hip replacement," International Orthopaedics, vol. 40, no. 3, pp. 481-485, 2016.

[26] A. W. Bodrogi, R. Sciortino, D. A. Fitch, and W. Gofton, "Use of the supercapsular percutaneously assisted total hip approach for femoral neck fractures: surgical technique and case series," Journal of Orthopaedic Surgery and Research, vol. 11, no. 1, p. 113, 2016.

[27] H. Jiang, Clinic of Orthopedics, Tianxiang East Hospital, Yiwu, China, L.-H. Wang et al., "Supercapsular percutaneously assisted total hip arthroplasty versus conventional posterior approach: comparison of early functional results," Acta Orthopaedica et Traumatologica Turcica, vol. 54, no. 5, pp. 511-515, 2020.
[28] G. E. Lewinnek, J. L. Lewis, R. Tarr, C. L. Compere, and J. R. Zimmerman, "Dislocations after total hip-replacement arthroplasties," The Journal of Bone and Joint Surgery. American Volume, vol. 60, no. 2, pp. 217-220, 1978.

[29] H. L. M. Williams, G. E. Bartlett, M. R. Norton, and R. G. Middleton, "The posterior acetabular wall (PAW): an aid to acetabular orientation at primary THA," Hip International, vol. 28, no. 1, pp. 29-32, 2018. 